\title{
ARTICLES
}

\section{Atomic supersymmetry and the Stark effect}

\author{
Robert Bluhm \\ Physics Department, Colby College, Waterville, Maine 04901 \\ V. Alan Kostelecký \\ Physics Department, Indiana University, Bloomington, Indiana 47405
}

(Received 27 May 1992)

\begin{abstract}
A search is conducted for physical quantum-mechanical supersymmetries involving the hydrogen atom. In all coordinate systems admitting a direct separation of the Schrödinger equation, the structure of the separated equations is examined for possible supersymmetric extensions. In addition to the known supersymmetry involving the radial equation for spherical coordinates, we uncover a related supersymmetry involving the radial equation for conical coordinates and a pair of supersymmetries involving parabolic coordinates. The associated spectra and possible physical import of the latter are discussed. They connect certain eigenfunctions of the hydrogen and lithium atoms in the unbroken-symmetry limit. Following the established procedure for the case of spherical coordinates, the breaking of these parabolic supersymmetries is incorporated in a model constructed using notions of quantum-defect theory. The model yields analytical wave functions in parabolic coordinates for the valence electron of alkali-metal atoms, while correctly reproducing the eigenvalue spectra. These ideas are applied to the study of the Stark effect in alkali-metal atoms. Using supersymmetry-based quantum-defect eigenfunctions, we obtain Stark maps for lithium and sodium. The spherical case shows striking agreement with experiment.
\end{abstract}

PACS number(s): 03.65.Fd, 03.65.Ge, 11.30.Pb, 31.15.+q

\section{INTRODUCTION}

The physics of most atomic systems is too complex to admit direct analytical solution. To address such issues, enough approximations must be made to render the problem tractable either to analytical or to numerical methods. Exact or broken symmetries often provide a useful guide to the choice of approximation, while permitting physical insight to be maintained. The search for symmetries is evidently a crucial part of this procedure.

The behavior of a highly excited valence electron of an atom or ion can be approximated as that of a single electron in an effective central potential [1]. This viewpoint is particularly useful for cases where the valence electron is the sole one outside a closed shell, as occurs in alkalimetal atoms. Several methods exist for calculating the effective potential [2], and the physics of such situations continues to attract interest [3].

A realistic central-potential approximation for the valence electron of an atom or ion can be interpreted as the implementation of a broken symmetry [4]. In the limit that the central potential is hydrogenic, the symmetry is exact. It then connects the atom under study to the hydrogen atom, mathematically linking their eigenvalues and eigenfunctions while implementing the Pauli principle for the valence electron. The symmetry is broken by nonhydrogenic contributions to the effective potential yielding, for example, the Rydberg series for alkali-metal atoms $[5,6]$. Nonetheless, it can be used as a guide for the construction of a central-potential model [7] reproducing the Rydberg series. The model incorporates notions of quantum-defect theory [8-10] but is motivated by symmetry considerations rather than phenomenology. It provides analytical eigenfunctions for the valence electron while maintaining features characteristic of the symmetry. Comparisons of the model's predicted transition probabilities for alkali-metal atoms [7] and alkalineearth-metal ions [11] with accepted values [12] show good agreement. The model's range of validity extends to reproducing some of the gross features of the finestructure of alkali-metal atoms [13]. The eigenfunctions have also been used as trial wave functions for detailed numerical calculations [14].

The symmetry in question, called quantum-mechanical supersymmetry [15], is based on an extension of ordinary Lie algebras to superalgebras, which incorporate anticommuting generators. Supersymmetries have found widespread application in many areas of physics [16]. In the central-potential context, the supersymmetry arises in the radial equation appearing upon the separation of the Schrödinger equation in spherical coordinates. Some background material on supersymmetric quantum mechanics and its use in the central-potential approximation is summarized in Sec. II of this paper [17]. 
In the present work, we undertake a systematic search for other quantum-mechanical supersymmetries associated with the hydrogen-atom Schrödinger equation. In Sec. III, we review all coordinate systems for which the problem is directly separable, i.e., without modulation factors. In Sec. IV, we then examine these systems to determine which can act as part of a supersymmetric multiplet. We thereby uncover a supersymmetry in conical coordinates and two supersymmetries in parabolic coordinates. The latter are physically distinct from the previously studied supersymmetry in spherical coordinates. Their possible physical relevance to the spectra and eigenfunctions of the alkali-metal atoms in the exact symmetry limit is discussed in Sec. V.

Symmetry-breaking effects for the parabolic supersymmetries can be introduced in a central-potential model that uses quantum defects to reproduce the Rydberg series and generates analytical eigenfunctions for the valence electron. The model incorporates many of the features of supersymmetry. It is presented in Sec. VI.

Since the breaking of supersymmetry is due to the valence electron interacting with the core, all the supersymmetry-based models are expected to work best for highly excited, or Rydberg, atoms. These have a wealth of interesting properties, and their structure in electric and magnetic fields is a subject of current experimental research $[3,18]$. In this paper, we focus on the Stark effect [19] as a means of testing the various models. In parabolic coordinates, the Stark problem for hydrogen is separable. Perturbation theory can be carried out to high orders [20,21] and compared to experiment [22]. However, for alkali-metal atoms, quantum defects appear. The Stark structure of Rydberg states for alkalimetal atoms has been studied both experimentally and numerically [23-25]. Section II D contains some background material on this topic. In Sec. VII, we use the analytical wave functions of the parabolic and spherical quantum-defect theories to generate Stark maps for Rydberg states of lithium and sodium. A summary and our conclusions are presented in Sec. VIII.

\section{BACKGROUND}

In this section, we provide a summary describing supersymmetric quantum mechanics, a brief review of prior results in atomic supersymmetry and in supersymmetrybased quantum-defect theory, and a short description of the standard approach to the Stark effect.

\section{A. Supersymmetric quantum mechanics}

If the Hamiltonian of a quantum-mechanical system can be expressed in terms of anticommuting generators $Q_{i}$ as $\left\{Q_{i}, Q_{j}\right\}=\delta_{i j} H$, with $\left[Q_{i}, H\right]=0$ for $i=1,2, \ldots, N$, then the system is said to be supersymmetric [15]. The superalgebra defined by these relations is denoted $\operatorname{sqm}(N)$. For the special case $\operatorname{sqm}(2)$, the linear combinations $Q=\left(Q_{1}+i Q_{2}\right) / \sqrt{2}$ and $Q^{\dagger}=\left(Q_{1}-i Q_{2}\right) / \sqrt{2}$ obey the relations

$$
H=\left\{Q, Q^{\dagger}\right\}, \quad[H, Q]=\left[H, Q^{\dagger}\right]=0
$$

In the simplest realization, the Hamiltonian for $\operatorname{sqm}(2)$ may be written as $H=H_{+} \oplus H_{-}$, where the components $H_{+}$and $H_{-}$containing the supersymmetric potential partners $V_{+}$and $V_{-}$are given by

$$
\begin{aligned}
& H_{ \pm} \Psi_{ \pm n}=\left[-\frac{d^{2}}{d x^{2}}+V_{ \pm}(x)\right] \Psi_{ \pm n}=\epsilon_{n} \Psi_{ \pm n}, \\
& V_{+}(x)=\left(\frac{1}{2} U^{\prime}\right)^{2}-\frac{1}{2} U^{\prime \prime}, \quad V_{-}(x)=\left(\frac{1}{2} U^{\prime}\right)^{2}+\frac{1}{2} U^{\prime \prime} .
\end{aligned}
$$

Here the prime means $d / d x$, and $U(x)$ is a function that depends on the problem being studied. Except for the ground-state eigenvalue, which is associated with $\boldsymbol{H}_{+}$ only, the two Hamiltonians have the same spectra. We refer to the eigenspectra of $H_{+}$and $H_{-}$as the bosonic and fermionic stacks, respectively.

The Hamiltonians $\boldsymbol{H}_{+}$and $\boldsymbol{H}_{-}$can be written as $H_{+}=A^{\dagger} A$ and $H_{-}=A A^{\dagger}$, where

$$
\begin{aligned}
& A=-i\left(\partial_{x}+\frac{1}{2} U^{\prime}\right), \\
& {\left[A, A^{\dagger}\right]=U^{\prime \prime} .}
\end{aligned}
$$

The supersymmetry generators $Q, Q^{\dagger}$ map degenerate states in the bosonic and fermionic stacks into one another. Explicitly, they are given as $2 \times 2$ matrices,

$$
Q=\left[\begin{array}{cc}
0 & 0 \\
A & 0
\end{array}\right], \quad Q^{\dagger}=\left[\begin{array}{cc}
0 & A^{\dagger} \\
0 & 0
\end{array}\right]
$$

and obey $Q^{2}=\left(Q^{\dagger}\right)^{2}=0$. The Hamiltonian is

$$
H=\left\{Q, Q^{\dagger}\right\}=\left(\begin{array}{cc}
H_{+} & 0 \\
0 & H_{-}
\end{array}\right)
$$

\section{B. Atomic supersymmetry}

The radial part of the hydrogen-atom Schrödinger equation expressed in spherical coordinates can be written as one of the two partner Hamiltonians for an $\operatorname{sqm}(2)$ supersymmetry [4]. It has the form

$$
\left[-\frac{d^{2}}{d y^{2}}-\frac{1}{y}+\frac{l(l+1)}{y^{2}}-\frac{1}{2} E_{n}\right] \chi_{n l}(y)=0,
$$

where $y=2 r, E_{n}=-1 / 2 n^{2}$, and $\chi_{n l}(2 r)=r R_{n l}(r)$. We use atomic units throughout this paper, but note that $y$ differs from $r$ by a factor of 2 . The radial wave functions are given by

$$
\begin{aligned}
R_{n l}(r)= & \frac{2}{n^{2}}\left[\frac{\Gamma(n-l)}{\Gamma(n+l+1)}\right]^{1 / 2}\left[\frac{2 r}{n}\right]^{l} \\
& \times \exp \left[-\frac{r}{n}\right] L_{n-l-1}^{(2 l+1)}\left[\frac{2 r}{n}\right],
\end{aligned}
$$

where the associated Laguerre polynomials are defined by [26]

$L_{n}^{(\alpha)}(x)=\sum_{p=0}^{n}(-x)^{p} \frac{\Gamma(n+\alpha+1)}{p ! \Gamma(p+\alpha+1) \Gamma(n-p+1)}$.

Equation (2.8) can be written in the supersymmetric form 
(2.2) for $H_{+}$by choosing

$$
U(y)=\frac{y}{l+1}-2(l+1) \ln y .
$$

The mathematical form of the supersymmetry partner to the hydrogen atom can be found by fixing $l$ and solving for $V_{-l}$ in Eq. (2.3). The supersymmetric eigenfunctions of $V_{-l}$ are $R_{n, l+1}$, with $n \geq 2$. They form a complete set.

Reference [4] argued on physical grounds that the $s$ levels of lithium may be interpreted as the supersymmetric partners of the hydrogen atom $s$ levels. The supersymmetry can be repeated, in the sense that the $s$ orbitals of lithium are in turn supersymmetric partners of the sodium $s$ orbitals. There are also connections among $p$ and higher orbitals. The characteristic feature of supersymmetry in this context is the integer shift in the quantum number $l$. It is intimately connected with the Pauli principle and the resulting exclusion of the lithium valence electron from the $1 s$ levels.

\section{Supersymmetry-based quantum-defect theory}

In alkali-metal atoms, the interactions between the valence electron and the electrons in the inner shells break the supersymmetry, and the energy levels become shifted relative to those of hydrogen. Mathematically, the change in energy can be described by introducing a quantum defect $\delta(n, l)[5]$ :

$$
\begin{aligned}
& n^{*}=n-\delta(n, l), \\
& E_{n}=-\frac{1}{2 n^{* 2}},
\end{aligned}
$$

where $\delta(n, l)$ is approximately constant for a given fixed orbital quantum number $l$. The exact quantum defects $\delta(n, l)$ rapidly approach asymptotic values $\delta(l)$ as $n$ increases.

Reference [7] combined supersymmetry ideas with the notion of quantum defects to incorporate supersymmetry-breaking effects arising from the presence of an effective potential for the valence electron. A new orbital quantum number,

$$
l^{*}=l-\delta(l)+I(l)
$$

is introduced, where $I(l)$ is an integer. The radial equation obtained by replacing $n, l, E_{n}$ by $n^{*}, l^{*}, E_{n} *$ defines a quantum-defect theory that has analytical solutions with eigenenergies given by the Rydberg series (2.13). It also takes into account the integer shifts in $l$ that are characteristic of supersymmetry. However, the supersymmetry is broken when $\delta(l) \neq 0$.

The eigenvectors of the quantum-defect theory are given by

$$
\begin{aligned}
R_{n^{*} l^{*}}(r)= & \frac{2}{n^{* 2}}\left[\frac{\Gamma\left(n^{*}-l^{*}\right)}{\Gamma\left(n^{*}+l^{*}+1\right)}\right]^{1 / 2}\left[\frac{2 r}{n^{*}}\right]^{l^{*}} \\
& \times \exp \left[-\frac{r}{n^{*}}\right] L_{n^{*}-l^{*}-1}^{\left(2 l^{*}+1\right)}\left(\frac{2 r}{n^{*}}\right) .
\end{aligned}
$$

nomials because $n^{*}-l^{*}-1=n-l-I(l)-1$ remains an integer. For asymptotic quantum defects $\delta(l)$, these eigenfunctions form an orthonormal and complete set.

\section{The Stark effect}

The valence electron of an alkali-metal atom in an external electric field can be described by the Hamiltonian

$$
H=-\frac{1}{2} \nabla^{2}+V(\mathbf{r})+F z,
$$

where $F$ is the magnitude of the electric field, taken to point in the $z$ direction. The form of $V(\mathbf{r})$ is sufficiently complex that $H$ is not separable, and analytical solutions cannot be found. Instead, approximation methods must be used.

One approach uses a hydrogenic approximation in the context of a quantum-defect theory. The radial matrix elements can be integrated numerically using a Coulomb potential and setting the energies equal to the Rydberg series $E_{n *}=-1 / 2 n^{* 2}$. The integration is performed inward from large values of $r$, and when the solution starts to diverge near the inner core, the integration is terminated. The resulting energy matrix, including the Stark term, is diagonalized for a subset of basis states. The eigenvalues are thereby generated as a function of the electric field.

In a classic paper [23], the Stark structures of Rydberg states of lithium were computed in this way and then compared to experimental measurements. For $n=15$ manifolds, diagonalization of a subset of states consisting of $n=13-20$ yielded numerical results in good agreement with the experimental Stark maps. Similar computations were carried out for sodium and other alkali-metal atoms.

\section{SEPARATION OF VARIABLES}

The laplacian operator is directly separable, i.e., without use of a modulation factor, in eleven different coordinate systems [27]. Only four of these separate the Schrödinger equation with a Coulomb potential $V(r)=-1 / r$ : spherical, conical, prolate spheroidal, and parabolic coordinates. This can be traced to the $O(4)$ symmetry of hydrogen [28]. Here, we review the separation of variables for the hydrogen atom in each of these coordinate systems.

\section{A. Spherical coordinates}

In spherical coordinates $r, \theta, \phi$, the Coulomb-problem eigenfunctions can be written

$$
\Psi_{n l m}(r, \theta, \phi)=R_{n l}(r) Y_{l m}(\theta, \phi) .
$$

The radial wave functions are given in terms of the associated Laguerre polynomials in Eq. (2.9), and the spherical harmonics are

$Y_{l m}(\theta, \phi)=\frac{1}{\sqrt{2 \pi}}\left[\frac{2 l+1}{2} \frac{(l-m) !}{(l+m) !}\right]^{1 / 2} P_{l}^{m}(\cos \theta) \Phi(\phi)$.

The solution still involves the associated Laguerre poly- 
The functions $P_{l}^{m}(\cos \theta)$ are the associated Legendre polynomials, which satisfy the equation

$\left(1-x^{2}\right)\left(P_{l}^{m}\right)^{\prime \prime}-2 x\left(P_{l}^{m}\right)^{\prime}+\left[l(l+1)-\frac{m^{2}}{1-x^{2}}\right] P_{l}^{m}=0$,

with $x=\cos \theta$. The equation for $\Phi$ is

$$
\frac{d^{2} \Phi}{d \phi^{2}}+m^{2} \Phi=0
$$

\section{B. Conical coordinates} [27]

The definitions of the conical coordinates $u, v, w$ are

$$
\begin{aligned}
& x^{2}=\left[\frac{u v w}{b c}\right]^{2}, \\
& y^{2}=\frac{u^{2}\left(v^{2}-b^{2}\right)\left(b^{2}-w^{2}\right)}{b^{2}\left(c^{2}-b^{2}\right)}, \\
& z^{2}=\frac{u^{2}\left(c^{2}-v^{2}\right)\left(c^{2}-w^{2}\right)}{c^{2}\left(c^{2}-b^{2}\right)},
\end{aligned}
$$

with $c^{2}>v^{2}>b^{2}>w^{2}$. The surfaces of constant $u, v, w$ are, respectively, spheres and cones of elliptic cross section pointing along the $z$ and $x$ axes. The conical coordinate $u$ is equal to the radial coordinate $r$ in spherical coordinates:

$$
u^{2}=x^{2}+y^{2}+z^{2}=r^{2} .
$$

The Coulomb problem separates in these coordinates, and the wave functions can be written

$$
\Psi_{n l \tau}=R_{n l}(r) \psi_{l \tau}(v, w)
$$

The radial functions $R_{n l}(r)$ are the same as in spherical coordinates. The functions $\psi_{l \tau}(v, w)$ are products of Lamé functions [29] and are eigenstates of the angular momentum, but with the angular momentum vector having unusual orientations. The quantum number $m$ is replaced by a number $\tau$, which designates the eigenvalues of angular-momentum vectors oriented along elliptic cones about the $x$ or $z$ axes.

Separating the functions $\psi_{l \tau}(v, w)$ gives

$$
\psi_{l \tau}(v, w)=\chi_{1}(v) \chi_{2}(w)
$$

where the equations for the Lamé functions are

$$
\begin{array}{r}
{\left[\left[\left(v^{2}-b^{2}\right)\left(c^{2}-v^{2}\right)\right]^{1 / 2} \frac{\partial}{\partial v}\left[\left[\left(v^{2}-b^{2}\right)\left(c^{2}-v^{2}\right)\right]^{1 / 2} \frac{\partial}{\partial v}\right]\right.} \\
\left.+l(l+1) v^{2}-\mu\right] \chi_{1}(u)=0
\end{array}
$$

$$
\begin{aligned}
& {\left[\left[\left(b^{2}-w^{2}\right)\left(c^{2}-w^{2}\right)\right]^{1 / 2} \frac{\partial}{\partial w}\right.} \\
& \times\left[\left[\left(b^{2}-w^{2}\right)\left(c^{2}-w^{2}\right)\right]^{1 / 2} \frac{\partial}{\partial w}\right] \\
& \left.-l(l+1) w^{2}+\mu\right] \chi_{2}(v)=0 .
\end{aligned}
$$

Here, $l(l+1)$ is the separation constant that is introduced when the radial wave function $R_{n l}(r)$ is separated, and $\mu$ is a second separation constant that is introduced when the $v$ and $w$ variables are separated.

\section{Prolate-spheroidal coordinates}

With $u, v, w$ defined by

$$
\begin{aligned}
& x=a \sinh u \sin v \cos w, \\
& y=a \sinh u \sin v \sin w, \\
& z=a \cosh u \cos v,
\end{aligned}
$$

the prolate-spheroidal coordinates $\xi, \eta, \phi$ are defined by [27]

$$
\begin{aligned}
& \zeta=\cosh u, \quad 1 \leq \zeta<\infty \\
& \eta=\cos v, \quad-1 \leq \eta \leq 1 \\
& \phi=w, \quad 0 \leq \phi \leq 2 \pi .
\end{aligned}
$$

Prolate-spheroidal coordinates are useful for problems having two centers of force, such as the $\mathrm{H}_{2}{ }^{+}$molecule. However, it has been shown that the hydrogen-atom problem is separable in prolate-spheroidal coordinates [30]. This is achieved by setting up the problem as if there were two centers of force with two nuclear charges, but then setting the magnitude of one of the charges equal to zero. If the wave function is written

$$
\Psi(\zeta, \eta, \phi)=X(\zeta) Y(\eta) \Phi(\phi),
$$

then the functions $X, Y$, and $\Phi$ must satisfy the equations

$$
\begin{aligned}
& \frac{d}{d \zeta}\left[\left(\xi^{2}-1\right) \frac{d X}{d \zeta}\right] \\
& +\left[A+R\left(Q_{1}+Q_{2}\right) \xi-p^{2} \xi^{2}-\frac{m^{2}}{\xi^{2}-1}\right] X=0, \\
& \frac{d}{d \eta}\left[\left(1-\eta^{2}\right) \frac{d Y}{d \eta}\right] \\
& -\left[A+R\left(Q_{1}-Q_{2}\right) \eta-p^{2} \eta^{2}+\frac{m^{2}}{1-\eta^{2}}\right] Y=0, \\
& \frac{d^{2} \Phi}{d \phi^{2}}+m^{2} \Phi=0 .
\end{aligned}
$$

Note that the solutions $X$ and $Y$ to Eqs. (3.14) and (3.15) are different, even though the form of the equations is identical, because the variables $\zeta$ and $\eta$ have different 
domains. The parameters $m$ and $A$ are separation constants. The quantities $Q_{1}$ and $Q_{2}$ are the two charges, separated by a distance $R$. For the hydrogen atom, we take $Q_{1}=1$ and $Q_{2}=0$. The constant $p$ is defined by $p^{2}=\frac{1}{2} E R^{2}$. Reference [28] shows that the allowed eigenenergies are the usual ones, $E=-1 / 2 n^{2}$.

\section{Parabolic coordinates}

Parabolic coordinates are defined [27] by

$$
\rho=r+z, \quad \sigma=r-z, \phi=\tan ^{-1}(y / x),
$$

where $r$ is the radial coordinate. The separation of variables for the Coulomb problem in parabolic coordinates can be written

$$
\Psi(\rho, \sigma, \phi)=u(\rho) v(\sigma) \Phi(\phi) .
$$

The $\Phi(\phi)$ equation is separated by introducing a nonnegative integer $m$, and the solutions are the usual azimuthal ones:

$$
\Phi(\phi)=e^{ \pm i m \phi} .
$$

The remaining two-dimensional equation can be written

$$
\begin{aligned}
\frac{1}{u} \frac{d}{d \rho}\left[\rho \frac{d u}{d \rho}\right]+ & \frac{1}{v} \frac{d}{d \sigma}\left[\sigma \frac{d v}{d \sigma}\right) \\
& -\frac{m^{2}}{4}\left(\frac{1}{\rho}+\frac{1}{\sigma}\right)+\frac{E}{2}(\rho+\sigma)+Q=0,
\end{aligned}
$$

where $Q$ is the nuclear charge, with $Q=1$ for hydrogen. For the $\rho, \sigma$ separation, two separation constants $Z_{1}, Z_{2}$ are introduced that obey

$$
Z_{1}+Z_{2}=Q
$$

The resulting separated equations for $u$ and $v$ are

$$
\begin{aligned}
& {\left[\frac{d}{d \rho}\left[\rho \frac{d}{d \rho}\right]-\frac{m^{2}}{4 \rho}+\frac{E}{2} \rho+Z_{1}\right] u(\rho)=0 .} \\
& {\left[\frac{d}{d \sigma}\left[\sigma \frac{d}{d \sigma}\right]-\frac{m^{2}}{4 \sigma}+\frac{E}{2} \sigma+Z_{2}\right] v(\sigma)=0,}
\end{aligned}
$$

Note that these two equations are linked by Eq. (3.21).

\section{SEPARABLE SUPERSYMMETRIES}

In this section, each of the separated equations for the hydrogen atom derived in Sec. III is examined to see if it can form part of an $\operatorname{sqm}(2)$ realization. To act as a supersymmetric Hamiltonian, a separated equation must have one of the two forms given in Eq. (2.2). Also, its solutions must give rise to an infinite tower of states labeled by the eigenvalue in (2.2) (which need not be the true, physical energy), and the potential must be of the form given in Eq. (2.3) for some function $U(x)$. We refer to any resulting supersymmetry as a separable supersymmetry.

\section{A. Spherical coordinates}

In spherical coordinates, a supersymmetry can be constructed using the radial equation [4], as summarized in Sec. II B. The equations for the angular variables $\theta$ and $\phi$ are given in Eqs. (3.3) and (3.4). The associated Legendre equation (3.3) can be expressed in the form of Eq. (2.2) with no linear derivative term by removing a modulation factor: $P_{l}^{m}(x)=\left[1 /\left(1-x^{2}\right)^{1 / 2}\right] f(x)$. The resulting equation does not have an eigenvalue representing an infinite tower of states. The eigenvalue of $f(x)$ is zero. No interesting supersymmetry structure is available. Also, Eq. (3.4) has zero potential. Thus, the only separable equation in spherical coordinates that admits an interesting $\operatorname{sqm}(2)$ supersymmetric partner is the radial equation.

\section{B. Conical coordinates}

Since the radial equation for $R_{n l}$ is the same in conical coordinates as in spherical coordinates, the same supersymmetry as in Sec. II B can be constructed. The interpretation of Ref. [4] can also be used. For example, we may again model the $s$ states of lithium as the superpartners of the $s$ states of hydrogen. The difference between this case and the spherical case is that the angular-momentum vector has unusual orientations in conical coordinates. There may be physical applications involving the hydrogen atom for which conical coordinates are useful. For most applications, however, we do not expect any advantage of conical over spherical coordinates. Note that for atoms in external fields, for which there is a well-defined $z$ axis, the radial-coordinate supersymmetry is best applied in spherical coordinates.

The remaining two separated equations in conical coordinates are Eqs. (3.9) and (3.10) for the Lamé functions $\chi_{2}(v) \chi_{3}(w)$. Rescaling the solution to Eq. (3.9) by a modulation factor

$$
\chi_{2}(v)=\left[\left(v^{2}-b^{2}\right)\left(c^{2}-v^{2}\right)\right]^{1 / 4} f(v),
$$

we obtain an equation for $f(v)$ of the form (2.2) with no linear derivative term:

$$
\left[-\frac{d^{2}}{d v^{2}}+V_{+}(v)\right] f(v)=0 .
$$

However, this equation has only a zero eigenvalue, and there is no infinite tower of states. The same result follows for Eq. (3.10). Hence, the equations for the conical coordinates $v$ and $w$ have no interesting associated $\operatorname{sqm}(2)$ supersymmetries.

\section{Prolate-spheroidal coordinates}

The wave function in prolate-spheroidal coordinates is $\Psi(\zeta, \eta, \phi)=X(\zeta) Y(\eta) \Phi(\phi)$, and the three separated equations are given in Eqs. (3.14)-(3.16). Using a modulated factor for $X$,

$$
X(\zeta)=\frac{1}{\left(\zeta^{2}-1\right)^{1 / 2}} f(\zeta),
$$

we obtain an equation for $f(\zeta)$ that is in the standard form (2.2), 


$$
\left[-\frac{d^{2}}{d \zeta^{2}}+V_{+}(\zeta)\right] f(\zeta)=0,
$$

but with only a zero eigenvalue. Again, there is no infinite tower of states. The equation for $Y(\eta)$ gives an equation of the same form as that for $X$, with the same result. The equation for $\Phi(\phi)$, Eq. (3.16), is already of the form (2.2) with an infinite tower of eigenvalues given by $m^{2}$. However, $V_{+}(\phi)=0$, so the superpartner equation also has a trivial potential $V_{-}(\phi)=0$. In summary, there is no interesting $\operatorname{sqm}(2)$ structure in prolate-spheroidal coordinates.

\section{Parabolic coordinates}

In parabolic coordinates, the wave functions are given by $\Psi(\rho, \sigma, \phi)=u(\rho) v(\sigma) \Phi(\phi)$. The $\Phi$ equation has zero eigenvalue, as usual. The remaining separated equations are Eqs. (3.22) and (3.23).

To rewrite Eq. (3.22) in the form required for supersymmetry, we rescale $u(\rho)$ by a modulation factor

$$
u(\rho)=\rho^{-1 / 2} \chi_{1}(\rho) .
$$

The resulting equation for $\chi_{1}$ is

$$
\left[\frac{d^{2}}{d \rho^{2}}+\frac{1-m^{2}}{4 \rho^{2}}+\frac{Z_{1}}{\rho}+\frac{E}{2}\right] \chi_{1}(\rho)=0 .
$$

This equation admits a supersymmetric partner. If we fix $m$ and $Z_{1}$ and define

$$
\begin{aligned}
& V_{+}(\rho)=\frac{m^{2}-1}{4 \rho^{2}}-\frac{Z_{1}}{\rho}+\frac{Z_{1}^{2}}{(m+1)^{2}}, \\
& \epsilon=\frac{E}{2}+\frac{Z_{1}^{2}}{(m+1)^{2}},
\end{aligned}
$$

then $\chi_{1}(\rho)$ obeys Eq. (2.2) with $H_{+}=-d^{2} / d \rho^{2}+V_{+}(\rho)$. We can solve Eq. (2.3) for $U(\rho)$ to get

$$
U(\rho)=\left[\frac{2 Z_{1}}{m+1}\right] \rho-(m+1) \ln \rho .
$$

A similar set of equations arises in the $\sigma$ coordinate, involving $v(\sigma), \chi_{2}(\sigma), Z_{2}$, and $U(\sigma)$.

The solutions for $H_{+}$are the usual wave functions in parabolic coordinates for the hydrogen atom [1]

$$
\begin{aligned}
u_{n_{1} n_{2} m}(\rho)= & {\left[\frac{1}{\pi}\right]^{1 / 4} \varepsilon^{m / 2+1}\left[\frac{\Gamma\left(n_{1}+1\right)}{\Gamma\left(n_{1}+m+1\right)}\right]^{1 / 2} } \\
& \times e^{-(\varepsilon / 2) \rho} \rho^{m / 2} L_{n_{1}}^{(m)}(\varepsilon \rho)
\end{aligned}
$$

where $\varepsilon=\sqrt{-2 E}$ and

$$
n_{1}=\frac{Z_{1}}{\varepsilon}-\frac{1}{2}(m+1) \text {. }
$$

For the wave functions to remain finite, $n_{1}$ must be a non-negative integer. The solutions $v_{n_{1} n_{2} m}(\sigma)$ for the $\sigma$ equation have a similar form, and their finiteness leads to the introduction of the second non-negative integer

$$
n_{2}=\frac{Z_{2}}{\varepsilon}-\frac{1}{2}(m+1) .
$$

In what follows, we take the three basic quantum numbers as $n_{1}, n_{2}$, and $m$. They are used to label the eigenfunctions $u(\rho)$ and $v(\sigma)$. The separation constants $Z_{1}$ and $Z_{2}=1-Z_{1}$ can then be written as

$$
\begin{aligned}
& Z_{1}=\frac{n_{1}+\frac{1}{2}(m+1)}{n_{1}+n_{2}+m+1}, \\
& Z_{2}=\frac{n_{2}+\frac{1}{2}(m+1)}{n_{1}+n_{2}+m+1} .
\end{aligned}
$$

By adding $n_{1}$ and $n_{2}$, we obtain the usual energy levels of hydrogen, $E_{n}=-1 / 2 n^{2}$, with

$$
n=n_{1}+n_{2}+m+1 \text {. }
$$

The superpartner to Eq. (4.6) also obeys Eq. (2.2) but with the potential

$$
\begin{aligned}
V_{-}(\rho) & =\left(\frac{1}{2} U^{\prime}\right)^{2}+\frac{1}{2} U^{\prime \prime} \\
& =\frac{(m+1)(m+3)}{4 \rho^{2}}-\frac{Z_{1}}{\rho}+\frac{Z_{1}^{2}}{(m+1)^{2}} .
\end{aligned}
$$

This has the same form as $V_{+}(\rho)$, but with $m \rightarrow m+2$ in the first term. Again, a similar expression occurs for the $\sigma$ equation.

With $m$ and $Z_{1}$ fixed, we can solve for the superpartner wave functions $u^{(-)}(\rho)$. For these solutions to remain finite, the quantity

$$
\frac{Z_{1}}{\varepsilon}-\frac{1}{2}(m+3)
$$

must be a non-negative integer. If we keep the definition of the integer $n_{1}$ as in Eq. (4.11), then the quantity in Eq. (4.17) is $\left(n_{1}-1\right)$. For this to be non-negative, we must now require that $n_{1} \geq 1$. This contains the supersymmetric information that the ground state of the bosonic stack has no superpartner, as described in Sec. II A. The supersymmetry therefore maps the quantum numbers $n_{1}$ and $m$ as $m \rightarrow m+2$ and $n_{1} \rightarrow n_{1}-1$. The requirement that $Z_{1}$ remain fixed defines the mapping on $n_{2}$ : we must also have $n_{2} \rightarrow n_{2}-1$. Note that under the combined supersymmetry transformation $n=n_{1}+n_{2}+m+1$ remains fixed, as expected for a transformation connecting degenerate states.

The wave functions for the superpartners $u^{(-)}(\rho)$ to the solutions $u(\rho)$ therefore have the same form as in Eq. (4.10), but with $m \rightarrow m+2, n_{1} \rightarrow n_{1}-1$, and $n_{2} \rightarrow n_{2}-1$ :

$$
u^{(-)}(\rho)=u_{n_{1}-1, n_{2}-1, m+2}(\rho), \quad n_{1} \geq 1 .
$$

Similarly, for the solution in the parabolic coordinate $\sigma$, we find that the superpartners $v^{(-)}(\sigma)$ to the solutions $v(\sigma)$ are given as

$$
v^{(-)}(\sigma)=v_{n_{1}-1, n_{2}-1, m+2}(\sigma), \quad n_{2} \geq 1 .
$$

With $m$ fixed, each superpartner equation has an eigenspectrum consisting of an infinite tower of states labeled 
by the quantum numbers

$$
E=-\frac{1}{2} \frac{Z_{1}^{2}}{\left[n_{1}+\frac{1}{2}(m+1)\right]^{2}}
$$

for the $\rho$ equation, and

$$
E=-\frac{1}{2} \frac{\left(1-Z_{1}\right)^{2}}{\left[n_{2}+\frac{1}{2}(m+1)\right]^{2}}
$$

for the $\sigma$ equation. Here both $n_{1}$ and $n_{2}$ range over the positive integers. If we use the definitions of $Z_{1}$ and $Z_{2}$ in Eqs. (4.13) and (4.14), then the two sets of energies in Eqs. (4.20) and (4.21) are equal to $E_{n}=-1 / 2 n^{2}$, with $n \geq 2$.

The above discussion defines two pairs of operators in the formalism of Sec. II A: $Q(\rho), H(\rho)$ and $Q(\sigma), H(\sigma)$. Each pair obeys the $\operatorname{sqm}(2)$ relations Eq. (2.1). The operators act on the wave functions $\chi_{1}(\rho) \chi_{2}(\sigma)$. Taken together, they generate a direct-sum supersymmetry algebra $\operatorname{sqm}(2) \oplus \operatorname{sqm}(2)$.

\section{E. Discussion}

The wave functions $\Psi_{n_{1} n_{2} m}$ of hydrogen in parabolic coordinates can be written as linear combinations of the spherical-coordinate wave functions $\Psi_{n l m}$, where the coefficients in the expansion are Clebsch-Gordan coefficients [31]. The question then naturally arises as to whether there is a relation between the supersymmetries in parabolic coordinates and the supersymmetry in spherical coordinates. In fact, the two supersymmetries are different because they involve different sets of states of the hydrogen atom.

To see this explicitly, note that in spherical coordinates $l$ is fixed. The fermionic stack is a tower of states all having the same $l$ value. The quantum number $m$ does not appear in the radial equation, so its value is fixed as well. In parabolic coordinates, we fix $m$, and two supersymmetries result. One has $n_{2}$ fixed, the other has $n_{1}$ fixed. If the fermionic stack with fixed $m$ and $n_{2}$ is expanded in terms of spherical wave functions, we get a superposition of different $l$ states that do not all belong to the same supersymmetry spectrum in the spherical case. Furthermore, the spherical harmonics $Y_{l m}$ are unaffected by the supersymmetry in spherical coordinates, whereas when the supersymmetric parabolic states are mapped back to spherical states, the $Y_{l m}$ are affected. This means the parabolic supersymmetries must describe distinct physical situations from the spherical case.

Even though the supersymmetries are different, it is interesting to note that all involve the Laguerre polynomials. Formally, they all can be mapped into the same set of equations. That is, the function $U(\rho)$ in Eq. (4.9) is mathematically equivalent to the function $U(y)$ in spherical coordinates in Eq. (2.11) under the mapping $\rho=y$, $m=2 l+1$, and $Z_{1}=1$.

In Sec. VII, developments based on the spherical and parabolic supersymmetries discussed above are used to model the Stark effect in alkali-metal atoms. However, the hydrogen atom with the Stark interaction included is also directly separable in parabolic coordinates [1]. It is then natural to ask whether this system has a supersymmetry. Each separated equation in parabolic coordinates can indeed by expressed in the form of Eq. (2.2) by dividing out by a modulation factor. A nontrivial eigenvalue, the physical energy $E$, does appear and labels an infinite tower of states. However, the equation that the potential $U(x)$ must satisfy in Eq. (2.3) is not readily solved. A power series solution for $U(x)$ can be shown to exist and can be used to find an expression for $V_{-}(x)$. The resulting equation for the supersymmetric partner, however, cannot be solved in a straightforward way. Furthermore, exact values for the Stark energies of hydrogen are not known. These facts make unlikely the prospect of finding a useful physical application involving a phenomenological supersymmetry. Instead, in Secs. VI and VII we focus on finding trial wave functions for the zero-field case and using them to obtain the Stark maps.

\section{ATOMIC SUPERSYMMETRIES IN PARABOLIC COORDINATES}

In this section, we discuss the identification of the supersymmetries in parabolic coordinates with physical systems.

\section{A. Parabolic spectroscopic notation}

It turns out that the parabolic supersymmetries naturally relate hydrogen to lithium. To permit a detailed description of this connection, we begin by defining a spectroscopic notation based on the parabolic quantum numbers $n_{1}, n_{2}, m$ that provides a labeling of the valenceelectron states for light alkali-metal atoms. This is needed because the degeneracy of the $n$ states is lifted in the alkali metals, so the quantum numbers $n_{1}, n_{2}, m$ of hydrogen are no longer good. The same situation occurs for the spherical case as well. The quantum numbers $n, l, m$ are only approximately valid, but, nonetheless, a spectroscopic notation based on hydrogen is used to designate the states of the alkali metals, which are labeled as $2 s, 2 p$, etc.

To define the spectroscopic notation for alkali-metal atoms using parabolic quantum numbers, first consider the states of hydrogen when the atoms are placed in a weak external electric field. For a fixed value of $m \geq 0$, the states at level $n$ split into a manifold of $(n-m)$ distinct levels. The lowest-energy sublevel, which we refer to as the red Stark state, has $n_{1}=0$ and $n_{2}=n-m-1$, while the highest-energy sublevel, or blue Stark state, has $n_{1}=n-m-1$ and $n_{2}=0$. The states in between are labeled sequentially from $n_{1}=0$ up to $n_{1}=n-m-1$, with $n_{2}$ decreasing as $n_{1}$ increases. This fanlike arrangement of states is a consequence of the linear Stark effect shifting the energies by $\frac{3}{2} F n\left(n_{1}-n_{2}\right)$ to first order in the field. Figure 1 represents the states of hydrogen in a small fixed external field, labeled with parabolic quantum numbers.

Light alkali-metal atoms in an external electric field exhibit Stark structure similar to that of hydrogen with two exceptions: the zero-field energies are not all degenerate, 


$$
\begin{aligned}
& \overline{n_{1}=2} n_{2}=0 \\
& \begin{array}{ll}
n_{1}=1 & n_{2}=1
\end{array} \\
& \overline{n_{1}=0} n_{2}=2 \\
& \begin{array}{ll}
n_{1}=1 & n_{2}=0 \\
\hline n_{1}=0 & n_{2}=1
\end{array} \\
& \overline{n_{1}=0} n_{2}=1 \quad \overline{n_{1}=0} \quad n_{2}=0 \\
& \begin{array}{ll}
\overline{n_{1}=1} & n_{2}=0 \\
\hline n_{1}=0 & n_{2}=1
\end{array} \quad \overline{n_{1}=0} n_{2}=0 \\
& \overline{n_{1}=0} \quad n_{2}=0 \\
& m=0 \\
& m= \pm 1 \\
& m= \pm 2
\end{aligned}
$$

FIG. 1. The states of hydrogen as designated by the parabolic-coordinate quantum numbers $n_{1}, n_{2}, m$.

and as the field increases the lines from different $n$ levels do not cross as they do for hydrogen. Nonetheless, for small external fields $\left(F \approx 2 \times 10^{-7}\right.$ a.u. for $\left.n=15\right)$, the lines fan out just as they do for hydrogen.

For a spectroscopic notation based on the parabolic quantum numbers $n_{1}, n_{2}, m$, we choose to designate the energy levels of light alkali-metal atoms by the corresponding hydrogenic values. Thus, for fixed $n$ and $m$, the lowest level is designated the $n_{1}=0, n_{2}=n-m-1$ state. The next is the $n_{1}=1$ state, and so on, up to $n_{1}=n-m-1, n_{2}=0$. We refer to the lowest and highest lines as red and blue, respectively. For zero external field, all the levels except the lowest-lying states (with nonvanishing quantum defects) are essentially degenerate. Assuming the Stark lines do not cross as the electric field increases from zero, the designations of the states in terms of $n_{1}, n_{2}, m$ is unambiguous. Only for those fields that appear to cross would there be a potential problem. This problem would also exist for the spectroscopic notation based on the spherical quantum numbers $n, l, m$. Given that the lines never actually cross, as required by the no-crossing theorems [32], then the designation of states is completely unambiguous.

\section{B. Atomic supersymmetry in parabolic coordinates}

To identify a physical interpretation for the supersymmetries uncovered in parabolic coordinates, we must first establish the full supersymmetric eigenspectrum. The supersymmetries per se provide only a few mild restrictions on the structure of the bosonic stack. Indeed, we are free to select any infinite set of states from the hydrogen atom, provided the states have different energy eigenvalues. The choice of an appropriate bosonic stack thus reduces to the specification of the augmentation of the principle quantum number $n=n_{1}+n_{2}+m+1$ in the three-dimensional space $\left(n_{1}, n_{2}, m\right)$. A curve in this space intersecting exactly once each plane of constant $n$ determines a particular bosonic stack.
The role of the supersymmetries is largely to determine the corresponding fermionic stack. Since there are two parabolic supersymmetry generators $Q(\rho)$ and $Q(\sigma)$, each bosonic stack provides three possible choices for the fermionic stack. These are given by the action of $Q(\rho)$, $Q(\sigma)$, or $Q(\rho) Q(\sigma)$ on the bosonic eigenfunctions. Even though the eigenvalues of the three choices are identical, the wave functions are not. Together with the freedom of choice of the curve that specifies the bosonic stack, these three possibilities for the fermionic stack establish the existence a priori of a threefold infinity of supersymmetric partner spectra involving the hydrogen energy levels.

To restrict the choice of the supersymmetric system, further conditions selected on physical grounds must be imposed. The following desiderata appear reasonable.

(a) The requirement of a nontrivial result imposes the condition that the supersymmetry partners are nonzero for all levels but the lowest.

(b) To incorporate stability of the ground state, the physical ground state $1 s$ of hydrogen must be identified with the lowest state of the bosonic stack.

(c) Since the mathematical eigenvalue of the supersymmetry turns out to be just the physical energy, it is natural to construct the bosonic stack by choosing one state from each energy level in hydrogen.

Condition (a) is self-evident. Although condition (b) is not strictly required by the mathematics, it would be somewhat unnatural to choose an unstable state as the ground state of a purportedly physical supersymmetric Hamiltonian. This condition was also imposed in the spherical case [4]. Condition (c) means that the curve in the three-dimensional space $\left(n_{1}, n_{2}, m\right)$ specifying the bosonic stack must intersect planes of constant $n$ at integer values of the coordinates $\left(n_{1}, n_{2}, m\right)$ for every value of $n$.

The requirements $(a)-(c)$ are sufficient and necessary conditions implying the uniqueness of the fermionic partner to a given bosonic stack. The simplest way to see the sufficiency is to note that the conditions allow the first-excited state in the bosonic stack to be one of only four eigenfunctions, $u_{100} v_{100}, u_{010} v_{010}$, or $u_{001} v_{001} e^{ \pm i m \phi}$. The point is that each of these is such that either $Q(\rho)$ or $Q(\sigma)$ vanishes. The necessity of each condition is straightforward to demonstrate by counterexample.

Since $n_{1}, n_{2}, m$ cannot take fractional values, augmenting $n$ by one unit as one steps up the stack [condition (c)] means only one of the three quantum numbers increases by one unit per step. There are then two natural (but not unique) choices for the bosonic stack satisfying all three desiderata. One is the set of states $n_{1} \geq 0, n_{2}=0, m=0$, i.e., all the blue lines of hydrogen. The other is the set $n_{1}=0, n_{2} \geq 0, m=0$, i.e., all the red lines. (The $n_{1}=0$, $n_{2}=0, m=0$ ground state is both blue and red since it is the only line in the $n=1$ manifold.) Note that the third natural candidate, the set $n_{1}=0, n_{2}=0, m \geq 0$, fails because both $Q(\rho)$ and $Q(\sigma)$ vanish on $u_{00 m} v_{00 m} e^{ \pm i m \phi}$.

Once the full supersymmetric spectrum is chosen, the remaining task is the identification of a physical system described by the fermionic stack. Here, we proceed by analogy with the spherical-coordinate case [4]. The key idea is to interpret the absence of a supersymmetric 
partner for the ground state of the bosonic stack as due to the action of the Pauli principle. Thus, the equation determining the fermionic stack is interpreted as a onebody model for the valence electron of a more complicated atom, for which the ground state is inaccessible to the valence electron due to the presence of other electrons and the Pauli principle.

Since the supersymmetry requires the bosonic and fermionic stacks to have degenerate eigenspectra, which is not the case in reality, this interpretation can only be strictly valid if one ignores the interactions producing the nonhydrogenic part of the effective potential for the valence electron. The interactions break the supersymmetry and must be taken into account in any attempt at a realistic model. This is the purpose of the supersymmetry-based models discussed in Sec. VI. In the present section, we neglect these effects.

Using the spectroscopic notation introduced in Sec. VA, we can explicitly identify the supersymmetric partners of hydrogen in parabolic coordinates. One natural phenomenological supersymmetry, obtained using the $\rho$-coordinate generator $Q(\rho)$, exists between the blue lines of hydrogen and the blue lines of lithium (designated by $m=0, n_{1} \geq 1, n_{2}=0$ ). These states are shown in Fig. 2 . Note that the $n_{1}=n_{2}=m=0$ level of lithium is effectively absent, as it is a filled inner shell.

The wave functions for the supersymmetric partners of hydrogen for the $\rho$ coordinate are given in Eq. (4.18). For the $m=0$ blue states of lithium in the exact symmetry limit, this gives $u_{n_{1}-1,-1,2}(\rho), n_{1} \geq 1$, as the $\rho$ coordinate wave functions. Note that $n_{2}=-1$ in the $\rho$ coordinate wave function still leads to finite-valued solutions, since the parameters for the associated Laguerre polynomials $L_{n_{1}}^{(m)}$ do not involve $n_{2}$. The $\rho$-coordinate wave functions are then multiplied by $v_{n_{1} 00}(\sigma)$ to give the complete parabolic wave functions.

Similarly, we can identify the natural supersymmetry arising from the supersymmetry generator $Q(\sigma)$. The supersymmetric partners in this case are the red lines of hy-

\begin{tabular}{ll}
\hline$n_{1}=4$ & $n_{1}=4$ \\
\hline$n_{1}=3$ & $n_{1}=3$ \\
\hline$n_{1}=2$ & $n_{1}=2$ \\
\hline$n_{1}=1$ & $n_{1}=1$ \\
$n_{1}=0$ & \\
Hydrogen & \\
$2=0$ m $=0)$ & Lithium
\end{tabular}

FIG. 2. The supersymmetry between the blue $\left(n_{2}=0\right)$ lines of hydrogen and the blue $\left(n_{2}=0\right)$ lines of lithium. drogen and the red lines of lithium (designated by $m=0$, $\left.n_{1}=0, n_{2} \geq 1\right)$. The $\sigma$-coordinate wave functions for lithium are $v_{-1, n_{2}-1,2}(\sigma), n_{2} \geq 1$. The complete parabolic wave functions are then obtained by multiplying by $u_{0 n_{2} 0}(\rho)$.

To summarize, the natural phenomenological supersymmetries for $m=0$ are between the blue states of hydrogen and the blue states of lithium and between the red states of hydrogen and the red states of lithium.

We can similarly construct supersymmetries between lithium and sodium. By taking the $m=0$ blue states of lithium to form the spectrum of $\mathrm{H}_{+}$, we obtain supersymmetric partners that are degenerate except for the $n_{1}=1$ ground state, which is excluded. The lowest level for the superpartners in this case is the $n_{1}=2$ level, which corresponds to the valence electron in sodium being excluded from both the $n=1$ and 2 levels. A supersymmetry between the red lines of lithium and the red lines of sodium can also be developed. These constructions generalize to other atoms and ions in the Periodic Table, in analogy with the spherical-coordinate supersymmetry [4].

\section{QUANTUM-DEFECT THEORIES IN PARABOLIC COORDINATES}

As long as the supersymmetries remain unbroken and the electric field is kept at zero, the energy levels of the supersymmetric partners are degenerate with the hydrogenic values. Nonhydrogenic interactions break the supersymmetry and lift the degeneracy, shifting the energy levels by quantum defects. In this section, we combine the notations of quantum-defect theory with those of supersymmetry in parabolic coordinates to obtain a class of one-electron central-potential models for the valence electron of light alkali-metal atoms.

The first step is to define the quantum defect for alkali-metal atoms in terms of the spectroscopic notation based on the parabolic quantum numbers $n_{1}, n_{2}, m$. The asymptotic quantum defect $\delta\left(n_{1}, m\right)$ now depends on both $n_{1}$ and $m$. Explicit values for lithium are $\delta(0,0)=0.4, \delta(0,1)=\delta(1,0)=0.05$, and $\delta\left(n_{1}, m \geq 2\right)=0$. These definitions hold for all $n_{2}$ provided $n=n_{1}+n_{2}+m+1 \geq 2$. Values for sodium are $\delta(0,0)=1.35, \quad \delta(0,1)=\delta(1,0)=0.859, \quad \delta(0,2)=\delta(1,1)$ $=\delta(2,0)=0.01$, and $\delta\left(n_{1}, m \geq 3\right)=0$. Here $n_{2}$ takes on all allowed values subject to $n=n_{1}+n_{2}+m+1 \geq 3$. The Rydberg eigenenergies are then given by

$$
E_{n} *=-1 / 2 n^{* 2}, n^{*}=n-\delta\left(n_{1}, m\right),
$$

as discussed in Sec. II C.

Our starting point for defining a quantum-defect theory in parabolic coordinates is the two-dimensional Eq. (3.20) with $Q=1$ giving the wave functions $u(\rho)$ and $v(\sigma)$. We now add an effective potential $V_{\text {eff }}(\rho, \sigma)$ to the left-hand side of Eq. (3.20). By definition, it is chosen so that (i) the resulting energy eigenvalues $E^{*}$ reproduce the Rydberg series Eq. (6.1), (ii) the new wave functions $u^{*}, v^{*}$ maintain an analytical form of the same general type as before, and (iii) there are integer shifts in the par- 
abolic quantum numbers incorporating supersymmetry ideas. This set of conditions is satisfied by the surprisingly simple choice

$V_{\mathrm{eff}}(\rho, \sigma)=\frac{m^{2}}{4}\left(\frac{1}{\rho}+\frac{1}{\sigma}\right)-\frac{1}{4}\left(\frac{m_{1}^{* 2}}{\rho}+\frac{m_{2}^{* 2}}{\sigma}\right)$,

as is shown below. Here, the quantities $m_{1}^{*}$ and $m_{2}^{*}$ are parameters in the model that are to be specified in terms of the parabolic quantum numbers, the quantum defect, and the supersymmetry integers.

When Eq. (6.2) is substituted into the modified form of Eq. (3.20) as specified above, the equation separates into two pieces. As for the hydrogenic case, we introduce the modulation factors

$$
\begin{aligned}
& u^{*}(\rho)=\rho^{-1 / 2} \chi_{1}^{*}(\rho), \\
& v^{*}(\sigma)=\sigma^{-1 / 2} \chi_{2}^{*}(\sigma) .
\end{aligned}
$$

The result is the pair of equations

$$
\begin{aligned}
& {\left[\frac{d^{2}}{d \rho^{2}}+\frac{1-m_{1}^{* 2}}{4 \rho^{2}}+\frac{Z_{1}^{*}}{\rho}+\frac{E^{*}}{2}\right] \chi_{1}^{*}(\rho)=0,} \\
& {\left[\frac{d^{2}}{d \sigma^{2}}+\frac{1-m_{2}^{* 2}}{4 \sigma^{2}}+\frac{Z_{2}^{*}}{\sigma}+\frac{E^{*}}{2}\right] \chi_{2}^{*}(\sigma)=0 .}
\end{aligned}
$$

The separation constants $Z_{1}^{*}$ and $Z_{2}^{*}$ satisfy the constraint

$$
Z_{1}^{*}+Z_{2}^{*}=1 \text {. }
$$

For Eqs. (6.5) and (6.6) to have finite solutions, the quantities

$$
\begin{aligned}
& n_{1}^{*}=\frac{Z_{1}^{*}}{\sqrt{-2 E^{*}}}-\frac{1}{2}\left(m_{1}^{*}+1\right), \\
& n_{2}^{*}=\frac{Z_{2}^{*}}{\sqrt{-2 E^{*}}}-\frac{1}{2}\left(m_{2}^{*}+1\right)
\end{aligned}
$$

must be non-negative integers. Adding these equations and using Eqs. (6.1) and (6.7), we find

$$
\begin{aligned}
& n^{*} \equiv n-\delta=n_{1}^{*}+n_{2}^{*}+\frac{1}{2}\left(m_{1}^{*}+m_{2}^{*}\right)+1, \\
& Z_{1}^{*}=\frac{n_{1}^{*}+\frac{1}{2}\left(m_{1}^{*}+1\right)}{n^{*}}, \\
& Z_{2}^{*}=1-Z_{1}^{*}=\frac{n_{2}^{*}+\frac{1}{2}\left(m_{2}^{*}+1\right)}{n^{*}} .
\end{aligned}
$$

The form of Eq. (6.5) is that of Eq. (4.6), and the solutions can be written down directly by appropriate tran-

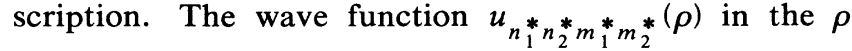
variable now depends on four quantum numbers. It is given by

$$
\begin{aligned}
& u_{n_{1}^{*} n_{2}^{*} m_{1}^{*} m_{2}^{*}}(\rho)=\left(\frac{1}{\pi}\right)^{1 / 4}\left(n^{*}\right)^{-\left(m_{1}^{*} / 2+1\right)} \\
& \times\left[\frac{\Gamma\left(n_{1}^{*}+1\right)}{\Gamma\left(n_{1}^{*}+m_{1}^{*}+1\right)}\right]^{1 / 2} \\
& \times e^{-\rho / 2 n^{*}} \rho^{m_{1}^{*} / 2} L_{n_{1}^{*}}^{\left(m_{1}^{*}\right)}\left(\frac{\rho}{n^{*}}\right) \text {. }
\end{aligned}
$$

Existence imposes the constraint $m_{1}^{*}>-1$. The wave function $v_{n_{1}^{*} n_{2}^{*} m_{1}^{*} m_{2}^{*}}(\sigma)$ solving Eqs. (6.4) and (6.6) is given by a similar expression, with $m_{2}^{*}, n_{2}^{*}$, and $\sigma$ replacing $m_{1}^{*}, n_{1}^{*}$, and $\rho$ on the right-hand side.

The wave functions for the full parabolic-coordinate quantum-defect theory are then

$$
\Psi_{n_{1} n_{2} m}(\rho, \sigma, \phi)=u_{n_{1}^{*} n_{2}^{*} m_{1}^{*} m_{2}^{*}}(\rho) v_{n_{1}^{*} n_{2}^{*} m_{1}^{*} m_{2}^{*}}(\sigma) e^{ \pm i m \phi},
$$

where on the left-hand side we have used spectroscopic notation to label the states. Note that $m$ is not shifted in the angular part of the solution in terms of $\phi$.

To make contact with the supersymmetry notions of Sec. IV D, we can define

$$
\begin{aligned}
& n_{1}^{*}=n_{1}-I_{1}\left(n_{1}, n_{2}, m\right), \quad n_{2}^{*}=n_{2}-I_{2}\left(n_{1}, n_{2}, m\right), \\
& m_{1}^{*}=m+\alpha_{1}\left(n_{1}, n_{2}, m\right), \quad m_{2}^{*}=m+\alpha_{2}\left(n_{1}, n_{2}, m\right),
\end{aligned}
$$

where $I_{1}$ and $I_{2}$ are integers. In terms of these four variables, the constraints arising from the requirement of existence of the eigenfunctions become

$$
I_{1} \leq n_{1}, \quad I_{2} \leq n_{2}, \quad \alpha_{1}>-m-1, \quad \alpha_{2}>-m-1,
$$

and

$$
\alpha_{1}+\alpha_{2}=2 I_{1}+2 I_{2}-2 \delta .
$$

In the limit of zero quantum defect $\delta$, Eq. (6.18) reduces the four variables $I_{1}\left(n_{1}, n_{2}, m\right), \quad I_{2}\left(n_{1}, n_{2}, m\right)$, $\alpha_{1}\left(n_{1}, n_{2}, m\right)$, and $\alpha_{2}\left(n_{1}, n_{2}, m\right)$ to three. For appropriate choices of these variables, the model then reduces to the supersymmetric case discussed in Secs. IVD and VB. For example, to obtain the supersymmetric wave functions $u_{n_{1}-1,-1,2}(\rho) v_{n_{1}, 00}(\sigma)$ describing the blue lines of lithium (spectroscopic notation $n_{1} \geq 1, n_{2}=0, m=0$ ) in the limit $\delta=0$, we choose $I_{1}\left(n_{1}, 0,0\right)=1, \alpha_{1}\left(n_{1}, 0,0\right)=2$, and all other values equal to zero. This gives

$$
\begin{aligned}
u_{n_{1}-1, n_{2}, m+2, m}^{*} & (\rho) v_{n_{1}-1, n_{2}, m+2, m}^{*}(\sigma) \\
& =u_{n_{1}-1, n_{2}-1, m+2}(\rho) v_{n_{1}, n_{2}, m}(\sigma),
\end{aligned}
$$

as needed. 
We have thus shown that the choice of Eq. (6.2) for the effective potential indeed satisfies the conditions (i)-(iii) stated at the beginning of this subsection. For fixed $\delta$, the result is a three-parameter family of supersymmetrybased quantum-defect theories in parabolic coordinates. Two parameters correspond to the presence of supersymmetry-type integer shifts in the quantum numbers $n_{1}$ and $n_{2}$. The third arises from the replacement of the azimuthal quantum number $m$ by two quantum numbers $m_{1}^{*}$ and $m_{2}^{*}$.

An important issue is the orthogonality of the model wave functions $\Psi_{n_{1} n_{2} m}$. For supersymmetric values of $I_{1}, I_{2}, \alpha_{1}, \alpha_{2}$, the wave functions are not fully orthogonal. There are nonzero off-diagonal matrix elements between states of different $n_{1}$ and $n_{2}$. Numerically, these are small, being less than a few percent of the diagonal contributions.

We have systematically sought values of the integers $I_{1}, I_{2}, \alpha_{1}, \alpha_{2}$ making the quantum-defect wave functions orthogonal, without success. One difficulty arises because the quantum defects $\delta\left(n_{1}, m\right)$ depend explicitly on both $n_{1}$ and $m$. For fixed $m$, the quantum defects can differ for different values of $n_{1}$, and as a result the states are not orthogonal in $n_{1}$.

In contrast, the spherical-coordinate quantum-defect wave functions $R_{n^{*}{ }^{*}}(r) Y_{l m}(\theta, \phi)$ discussed in Sec. II C are orthogonal for asymptotic values of the quantum defects [7]. Although the asymptotic quantum defects $\delta(l)$ depend explicitly on $l$, states of different $l$ are orthogonal because the spherical harmonics are orthogonal in $l$.

\section{THE STARK EFFECT}

In this section, we use the supersymmetry-based quantum-defect theories defined in parabolic and spherical coordinates to determine Stark maps.

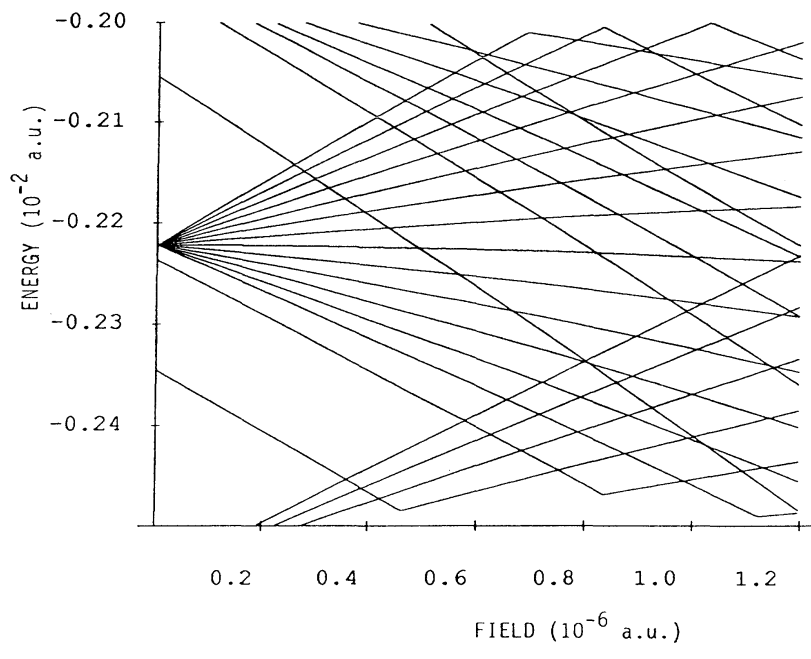

FIG. 3. Stark states of lithium for $m=0$ calculated using the quantum-defect theory defined in parabolic coordinates. The central manifold of lines represents $n=15$ states. The energy and field strength are in atomic units.

\section{A. Stark effect in parabolic coordinates}

We calculate the Stark states of alkali-metal atoms using the supersymmetry-based quantum-defect theory in parabolic coordinates. The zero-field wave functions are $\Psi_{n_{1} n_{2} m}(\rho, \sigma, \phi)=u_{n_{1}^{*} n_{2}^{*} m_{1}^{*} m_{2}^{*}}(\rho) v_{n_{1}^{*} n_{1}^{*} m_{1}^{*} m_{2}^{*}}(\sigma) e^{ \pm i m \phi}$,

where $u(\rho)$ is given in Eq. (6.13). We take from Sec. VI the values for the quantum defects for lithium and for the parameters $I_{1}, I_{2}, \alpha_{1}, \alpha_{2}$ corresponding to the supersymmetry of the $m=0$ blue lines.

In determining the Stark energies, we ignore the small off-diagonal components of the zero-field energies and obtain the matrix elements of the Stark corrections using the states in (7.1) as a basis:

$$
\begin{aligned}
& \iiint d \rho d \sigma d \phi \frac{1}{8}\left(\rho^{2}-\sigma^{2}\right) F \Psi_{n_{1 f} n_{2 f} m_{f}}^{*} \Psi_{n_{1 i} n_{2 i} m_{i}} \\
&=\frac{\pi F}{4}\left[\left\langle n_{1 f}^{*} n_{2 f}^{*} m_{1 f}^{*} m_{2 f}^{*}\left|\rho^{2}\right| n_{1 i}^{*} n_{2 i}^{*} m_{1 i}^{*} m_{2 i}^{*}\right\rangle\left\langle n_{1 f}^{*} n_{2 f}^{*} m_{1 f}^{*} m_{2 f}^{*} \mid n_{1 i}^{*} n_{2 i}^{*} m_{1 i}^{*} m_{2 i}^{*}\right\rangle\right. \\
&\left.\quad-\left\langle n_{1 f}^{*} n_{2 f}^{*} m_{1 f}^{*} m_{2 f}^{*} \mid n_{1 i}^{*} n_{2 i}^{*} m_{1 i}^{*} m_{2 i}^{*}\right\rangle\left\langle n_{1 f}^{*} n_{2 f}^{*} m_{1 f}^{*} m_{2 f}^{*}\left|\sigma^{2}\right| n_{1 i}^{*} n_{2 i}^{*} m_{1 i}^{*} m_{2 i}^{*}\right\rangle\right] .
\end{aligned}
$$

To calculate the parabolic matrix elements, we use the definition of the associated Laguerre polynomials given in Eq. (2.10) and we perform the integration directly [7]:

$$
\begin{aligned}
& \left\langle n_{1 f}^{*} n_{2 f}^{*} m_{1 f}^{*} m_{2 f}^{*}\left|\rho^{d}\right| n_{1 i}^{*} n_{2 i}^{*} m_{1 i}^{*} m_{2 i}^{*}\right\rangle \\
& =\frac{2}{\sqrt{\pi}}\left[\frac{\Gamma\left(n_{1 i}^{*}+1\right) \Gamma\left(n_{1 f}^{*}+1\right)}{\Gamma\left(n_{1 i}^{*}+m_{1 i}^{*}+1\right) \Gamma\left(n_{1 f}^{*}+m_{1 f}^{*}+1\right)}\right]^{1 / 2} \\
& \quad \times \sum_{p=0}^{n_{1 i}^{*}} \sum_{q=0}^{n_{1 f}^{*}} \frac{(-1)^{p+q}}{p ! q !} \frac{\left(2 n_{i}^{*}\right)^{m_{1 f}^{*} / 2+q+d}\left(2 n_{f}^{*}\right)^{m_{1 i}^{*} / 2+p+d}}{\left(n_{i}^{*}+n_{f}^{*}\right)^{(1 / 2)\left(m_{1 i}^{*}+m_{1 f}^{*}\right)+p+q+d+1}} \\
& \quad \times\left[\begin{array}{c}
n_{1 i}^{*}+m_{1 i}^{*} \\
m_{1 i}^{*}+p
\end{array}\right]\left[\begin{array}{c}
n_{1 f}^{*}+m_{1 f}^{*} \\
m_{1 f}^{*}+q
\end{array}\right) \Gamma\left[\frac{1}{2}\left(m_{1 i}^{*}+m_{1 f}^{*}\right)+p+q+d+1\right] .
\end{aligned}
$$


Here, $d$ is a positive integer, $n_{i}^{*}$ and $n_{f}^{*}$ are defined in Eq. (6.10), and $\left(\begin{array}{c}a \\ b\end{array}\right)=\Gamma(a+1) / \Gamma(b+1) \Gamma(a-b+1)$. The $\sigma$-space matrix elements obey a similar expression, but with $n_{1 i}^{*}$, etc., replaced by $n_{2 i}^{*}$, etc., on the right-hand side of Eq. (7.3). The above expressions can be evaluated numerically to any desired accuracy. For our calculations, we take the matrix elements $\left\langle n_{1 f}^{*} n_{2 f}^{*} m_{1 f}^{*} m_{2 f}^{*}\left|\rho^{d}\right| n_{1 i}^{*} n_{2 i}^{*} m_{1 i}^{*} m_{2 i}^{*}\right\rangle$ computed to eight significant digits.

The resulting energy matrix can be diagonalized for the subset of states with $n=13-17$ and the eigenvalues for the $n=15, m=0$ lines found as a function of the field strength. The ensuing Stark map is shown in Fig. 3. As can be seen, the curves are primarily linear and there are no quadratic Stark contributions. If we compare Fig. 3 with experimental measurements from Ref. [23], we see that for the parabolic quantum-defect theory the lines come much closer to crossing, and the characteristic large anticrossings are absent.

Other choices for the parameters $I_{1}, I_{2}, \alpha_{1}, \alpha_{2}$ yield similar maps. The parabolic quantum-defect theory does not yield Stark maps for alkali-metal atoms that are in good agreement with experimental results. The reason is that the basis of states used in the diagonalization of the energy matrix is not an orthogonal set.

\section{B. Stark effect in spherical coordinates}

We also used the supersymmetry-based quantumdefect theory to calculate the Stark states of alkali-metal atoms. The wave functions for zero field are given by

$$
\Psi_{n l m}(r, \theta, \phi)=R_{n} *_{l} *(r) Y_{l m}(\theta, \phi),
$$

where $R_{n * l^{*}}(r)$ is defined in Eq. (2.15). The $Y_{l m}(\theta, \phi)$ are the usual spherical harmonics. For lithium, the asymptotic values of $\delta(l)$ are [33] $\delta(0)=0.4, \delta(1)=0.05$, and $\delta(l \geq 2)=0$. The integers $I(l)$ are set equal to the values corresponding to the supersymmetric solutions [4]:

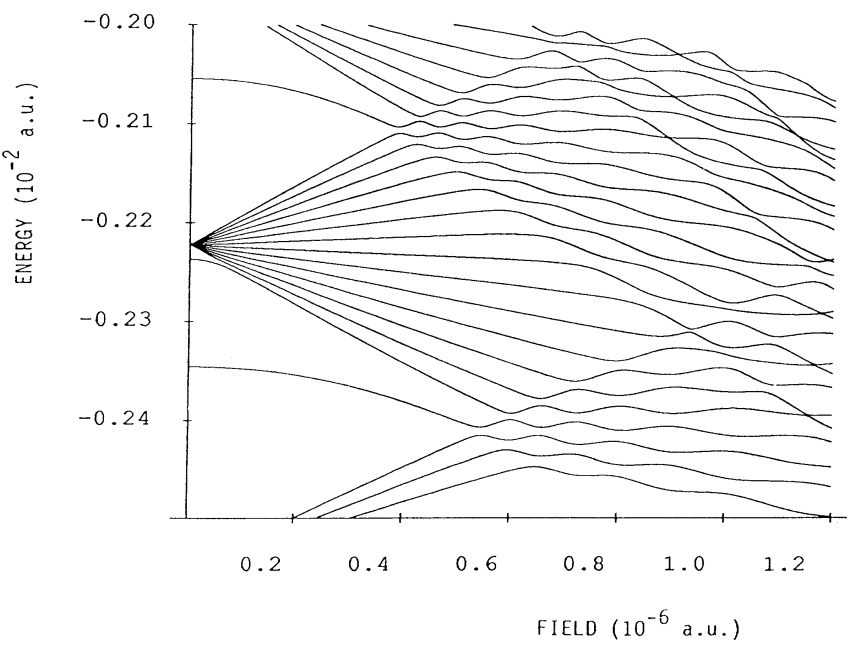

FIG. 4. Stark states of lithium for $n=15$ and $m=0$ calculated using the quantum-defect theory defined in spherical coordinates.

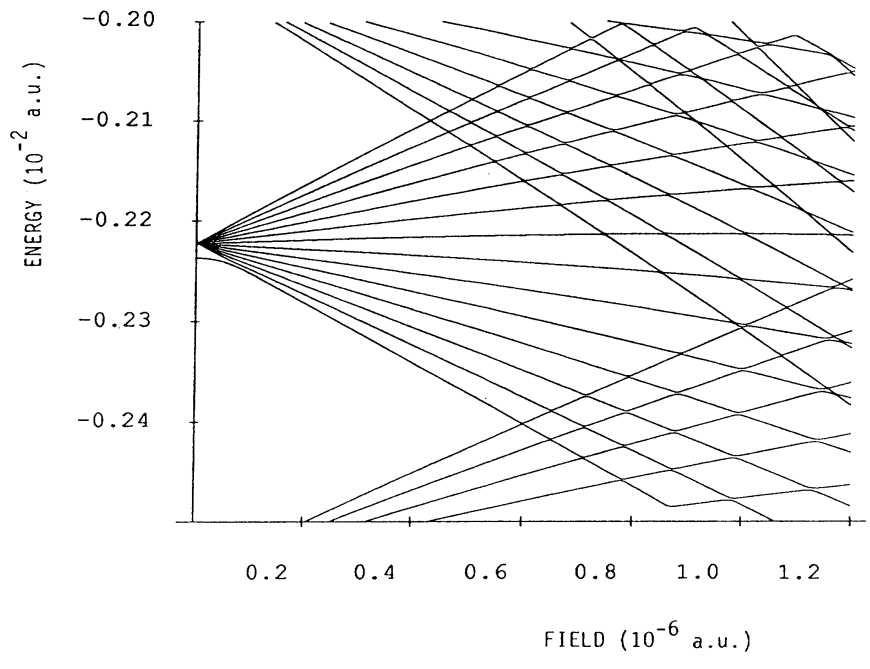

FIG. 5. Stark states of lithium for $n=15$ and $m=1$ calculated using the quantum-defect theory defined in spherical coordinates.

$I(0)=1$ and $I(l \geq 1)=0$. For sodium, $\delta(0)=1.35$, $\delta(1)=0.859, \delta(2)=0.01$, and $\delta(l \geq 3)=0$. The integers $I(l)$ in this case are $I(0)=2, I(1)=1$, and $I(l \geq 2)=0$.

We use the zero-field wave functions to calculate matrix elements of the Stark interaction. Since we are interested in obtaining the Stark maps of light alkali-metal atoms for $n=15$ states and for electric fields up to the approximate threshold limit near $1.2 \times 10^{-6}$ a.u. $\equiv 6.2$ $\mathrm{kV} / \mathrm{cm}$, we can neglect fine-structure effects.

The matrix elements of the Stark interaction take the form

$$
\begin{aligned}
& \left\langle n_{f}^{*} l_{f}^{*} m_{f}|F z| n_{i}^{*} l_{i}^{*} m_{i}\right\rangle \\
& \quad=\delta_{m_{i}, m_{f}} \delta_{l_{f}, l_{i} \pm 1} F\left\langle l_{f} m_{f}|\cos \theta| l_{i} m_{i}\right\rangle\left\langle n_{f}^{*} l_{f}^{*}|r| n_{i}^{*} l_{i}^{*}\right\rangle .
\end{aligned}
$$

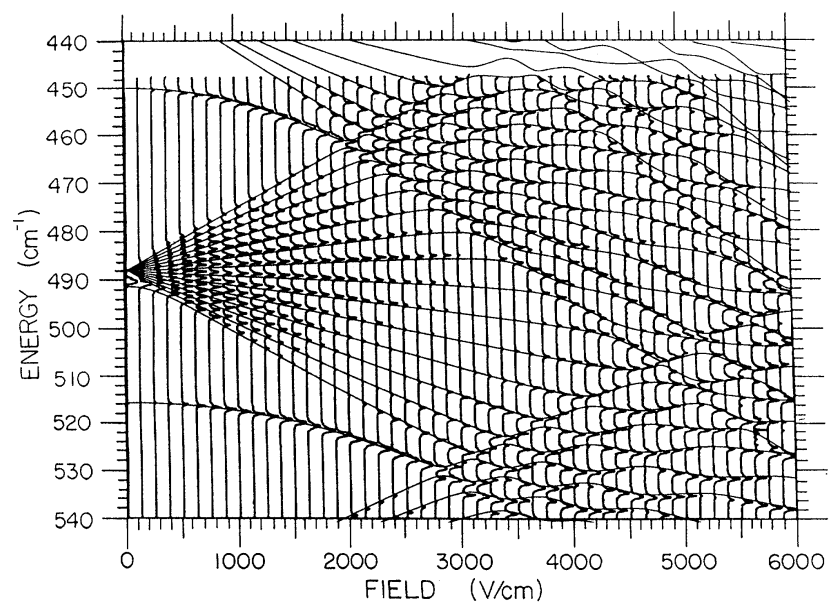

FIG. 6. Overlay of Fig. 4 with the experimental results reported in Ref. [23]. 


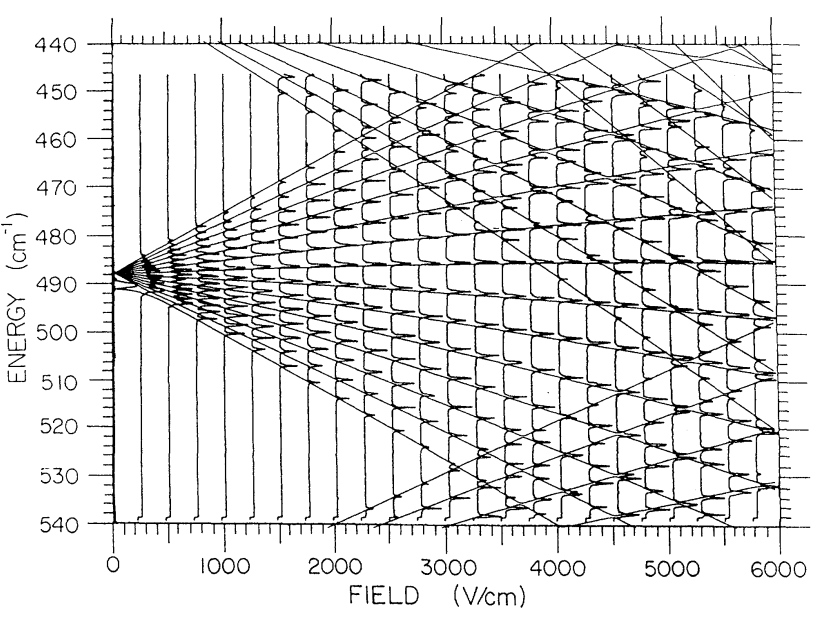

FIG. 7. Overlay of Fig. 5 with the experimental results reported in Ref. [23].

The angular integrations yield the results

$$
\begin{aligned}
& \left\langle l_{i} m_{i}|\cos \theta| l_{i}-1, m_{i}\right\rangle=\left(\frac{l_{i}^{2}-m_{i}^{2}}{\left(2 l_{i}+1\right)\left(2 l_{i}-1\right)}\right]^{1 / 2}, \\
& \left\langle l_{i} m_{i}|\cos \theta| l_{i}+1, m_{i}\right\rangle=\left[\frac{\left(l_{i}+1\right)^{2}-m_{i}^{2}}{\left(2 l_{i}+3\right)\left(2 l_{i}+1\right)}\right]^{1 / 2} .
\end{aligned}
$$

To calculate the radial matrix elements, we again use the form of the associated Laguerre polynomials given in Eq. (2.10), and we perform the integration of the radial matrix elements directly [7]. The resulting expression for $\left\langle n_{f}^{*} l_{f}^{*}|r| n_{i}^{*} l_{i}^{*}\right\rangle$ is exact. Again, we evaluate the matrix elements to eight significant digits.

The resulting energy matrix can be diagonalized for a subset of basis states. We examined the $n=15$ Stark states of lithium and sodium for both $m=0$ and 1 . We took as our basis of states the levels $n=13-20$. The results are displayed as a series of maps in Figs. 4-7. For comparison, we chose the range of energy and electric field used in Ref. [23]. The energies were calculated at $6.66 \times 10^{-9}$ a.u. $\equiv 32.4 \mathrm{~V} / \mathrm{cm}$ intervals, and the maps were generated by connecting the eigenvalues.

Figures 4 and 5 display our results for lithium. For $m=0$, the nonhydrogenic structure is readily apparent. There are sizable anticrossings, and the $s$ and $p$ states both display quadratic Stark effects for small values of the field. If Fig. 4 is compared with the numerical results of Ref. [23], it is seen that there are no discernible differences except for the $n=16$ lines at the top of the graph near the threshold limit (for $F$ near $1.2 \times 10^{-6}$ a.u.). The three apparent crossings noted in Ref. [23] are also visible in the upper part of Fig. 4 near $F=1.0 \times 10^{-6}$ a.u. For ease of comparison, Fig. 6 shows a superposition of Fig. 4 with the experimental data presented in Ref. [23]. The $m=1$ graph in Fig. 5 is more hydrogenlike in appearance than Fig. 4, since the only nonzero quantum defect in this case is quite small, $\delta(1)=0.05$. The anticrossings in this case are much smaller than for $m=0$. Figure 7 is a superposition of Fig. 5 with the experimen-

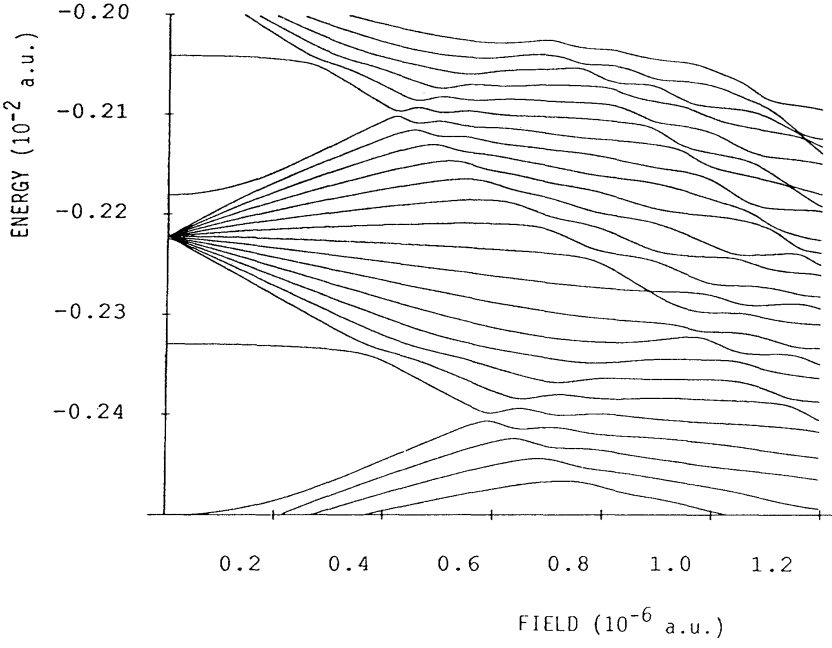

FIG. 8. Stark states of sodium for $n=15$ and $m=0$ calculated using the quantum-defect theory defined in spherical coordinates.

tal data of Ref. [23]. The agreement between our results and those of Ref. [23] is again quite striking. Some of the anticrossings in Fig. 5 are slightly smaller than those of Ref. [23], but this is probably because we plot three times as many points in making the graphs.

The graphs for sodium are shown in Figs. 8 and 9. For $m=0$ in Fig. 8, the quantum defects are quite large. The $16 s$ level is now below the $n=15$ manifold and is squeezed between the $15 p$ and $15 d$ lines. Likewise, the $15 s$ state falls below the $n=14$ lines and does not appear on the graph. There is an apparent crossing of the central $n=15$ lines near $F=9 \times 10^{-7}$ a.u. The $m=1$ graph in Fig. 9 is also nonhydrogenic in behavior. If the two graphs for sodium are compared with those computed numerically in Fig. [23], it is found that there are no no-

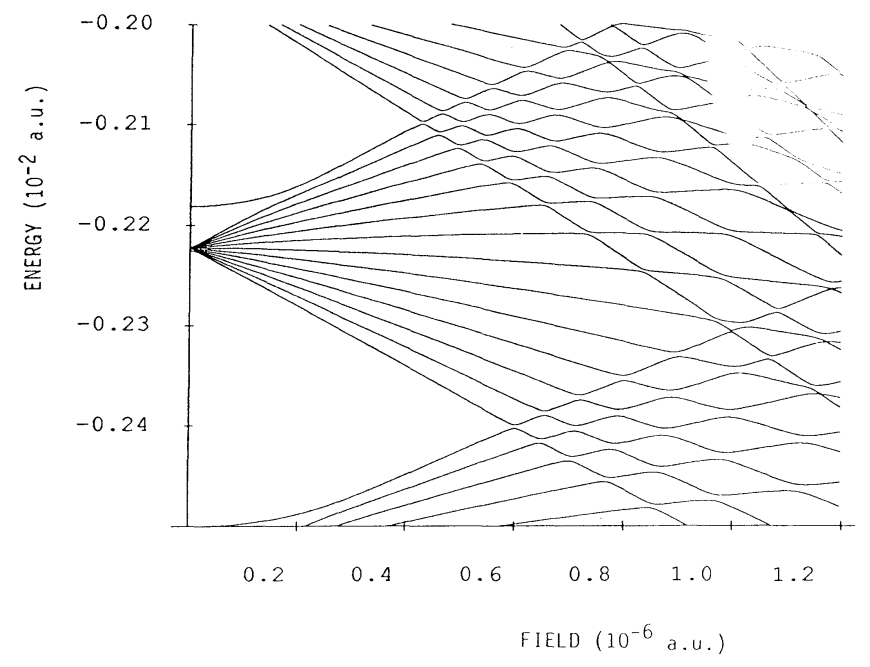

FIG. 9. Stark states of sodium for $n=15$ and $m=1$ calculated using the quantum-defect theory defined in spherical coordinates. 
ticeable differences except near the threshold limit for the uppermost lines. If we restrict our comparison to just the $n=15$ lines, then the two sets of lines are indistinguishable.

\section{SUMMARY}

In this paper, we presented a systematic study of quantum-mechanical supersymmetries involving the hydrogen atom. We treated all coordinates systems for which the Schrödinger equation is separable without the use of modulation factors. We found that the only separated equations that admit supersymmetric extensions are the radial equation in spherical coordinates, the radial equation in conical coordinates, and two of the equations in parabolic coordinates. The conicalcoordinate supersymmetry is the same as the previously known spherical-coordinate one. In contrast, the parabolic-coordinate supersymmetries contain different physics.

In a scenario approximating the behavior of the valence electron via a single-electron equation in an effective central potential, we established physical interpretations of the supersymmetries in the parabolic case. The exact-supersymmetry limit corresponds to a hydrogenic potential. The two most natural interpretations of the supersymmetries relate part of the spectrum and eigenfunctions of hydrogen to those of lithium in the exact-symmetry limit. In terms of the parabolic quantum numbers of hydrogen, $n_{1}, n_{2}, m$, the supersymmetries are found to occur for fixed $m$ and to be between the blue levels $\left(n_{2}=0\right)$ of hydrogen and the blue levels of lithium, or between the red levels $\left(n_{1}=0\right)$ of hydrogen and the red levels of lithium.

In a model based on notions of quantum-defect theory, we incorporated the supersymmetry breaking that arises from interactions generating a nonhydrogenic effective central potential. The model correctly reproduces the Rydberg series and yields analytical wave functions with supersymmetric features. However, unlike the eigenfunc- tions of spherical-coordinate quantum-defect theory in the limit of asymptotic quantum defects, the wave functions of the parabolic quantum-defect theory are not orthogonal. For lithium, the contributions to the matrix elements that generate violations of orthogonality are in general small, on the order of or less than a few percent of the diagonal elements.

One physical situation where the parabolic and spherical quantum-defect theories can be tested is the Stark effect. We calculated Stark maps of alkali atoms using both sets of quantum-defect theories. Making an approximation in the parabolic case, we found that the Stark maps for lithium $m=0$ states are primarily linear and lack the characteristic anti-crossings. This occurs because the corresponding wave functions do not form an orthogonal set.

In contrast, we showed that the spherical-coordinate quantum-defect theory yields Stark maps that are in striking agreement with experiment. This is in part a consequence of the orthogonality of the associated wave functions. Given the need for an approximation when parabolic coordinates are used, the spherical-coordinate wave functions appear more useful as trial wave functions for modeling the behavior of alkali-metal atoms.

\section{ACKNOWLEDGMENTS}

We enjoyed discussions with Charlie Conover, Mike Nieto, and Rod Truax. We thank Daniel Kleppner for permission to reproduce the experimental results of Ref. [23] in our Figs. 6 and 7. R.B. thanks Colby College for a Science Division Grant and the Theory Group at Indiana University for hospitality. V. A. K. thanks the Aspen Center for Physics and the Theory Division of CERN for hospitality. This research was supported in part by the United States Department of Energy under Contracts Nos. DE-AC02-84ER40125, Task B, and DEFG02-91ER40661, Task B.
[1] H. A. Bethe and E. Salpeter, Quantum Mechanics of Oneand Two-Electron Atoms (Plenum, New York, 1957).

[2] See, for example, E. U. Condon and G. H. Shortley, Theory of Atomic Spectra (Cambridge University Press, Cambridge, 1951); L. I. Schiff, Quantum Mechanics (McGraw-Hill, New York, 1955).

[3] Rydberg States of Atoms and Molecules, edited by R. F. Stebbings and F. B. Dunning (Cambridge University Press, Cambridge, 1983).

[4] V. A. Kostelecký and M. M. Nieto, Phys. Rev. Lett. 53, 2285 (1984); Phys. Rev. A 32, 1293 (1985).

[5] J. R. Rydberg, Kongl. Sven. Vetensk.-Akad. Hand. 23, no. 11 (1890); Philos. Mag. 29, 331 (1890).

[6] S. Bashkin and J. O. Stoner, Jr., Atomic Energy Levels and Grotrian Diagrams, Vol. 1 (Elsevier, New York, 1975).

[7] V. A. Kostelecký and M. M. Nieto, Phys. Rev. A 32, 3243 (1985).

[8] See, e.g., R. D. Cowan, The Theory of Atomic Structure and Spectra (University of California, Berkeley, 1981).
[9] C. H. Greene, U. Fano, and G. Strinati, Phys. Rev. A 19, 1485 (1979); C. H. Greene, A. Rau, and G. Strinati, ibid. 26, 2441 (1982); M. J. Seaton, Rep. Prog. Phys. 46, 167 (1983).

[10] See also G. Simons, J. Chem. Phys. 60, 645 (1974); V. A. Kostelecký and M. N. Nieto, Phys. Rev. A 43, 4063 (1991), and references therein.

[11] M. T. Djerad, J. Phys. II 1, 1 (1991).

[12] W. L. Wiese, M. W. Smith, and B. M. Glennon, Atomic Transition Probabilities, Vols. 1 and 2, Natl. Bur. Stand. (U.S.) Natl. Stand. Ref. Data Ser. Nos. 4 and 22 (U.S. GPO, Washington, DC, 1966 and 1969).

[13] V. A. Kostelecký, M. M. Nieto, and D. R. Truax, Phys. Rev. A 38, 4413 (1988).

[14] R. E. H. Clark and A. L. Merts, J. Quant. Spectrosc. Radiat. Transfer 38, 287 (1987).

[15] H. Nicolai, J. Phys. A 9, 1497 (1976); E. Witten, Nucl. Phys. B 188, 513 (1981).

[16] For an overview, see Supersymmetry in Physics, edited by 
V. A. Kostelecký and D. K. Campbell (North-Holland, Amsterdam, 1985).

[17] There is also a connection to parastatistics: J. Beckers and N. Debergh (unpublished).

[18] Atoms in Strong Fields, edited by C. A. Nicolaides and C. W. Clark (Plenum, New York, 1990).

[19] For a review, see K. J. Kollath and M. C. Standage, in Progress in Atomic Spectroscopy, Part B, edited by W. Hanle and H. Kleinpoppen (Plenum, New York, 1979).

[20] H. J. Silverstone, Phys. Rev. A 18, 1853 (1978); and in Ref. $[18]$.

[21] R. J. Damburg and V. V. Kolosov, in Rydberg States of Atoms and Molecules (Ref. [3]), p. 31.

[22] P. M. Koch, Phys. Rev. Lett. 41, 99 (1978); Atomic Physics (Plenum, New York, 1981), p. 181.

[23] M. L. Zimmerman, M. G. Littman, M. M. Kash, and D. Kleppner, Phys. Rev. A 20, 2251 (1979).

[24] D. Kleppner, M. G. Littman, and M. L. Zimmerman, in
Rydberg States of Atoms and Molecules (Ref. [3]), p. 73.

[25] T. F. Gallagher, in Progress in Atomic Spectroscopy, Part $D$, edited by $\mathbf{H}$. J. Beyer and H. Kleinpoppen (Plenum, New York, 1987).

[26] Handbook of Mathematical Functions, edited by $\mathbf{M}$. Abramowitz and I. A. Stegun (Dover, New York, 1965).

[27] P. M. Morse and H. Feshbach, Methods of Theoretical Physics, (McGraw-Hill, New York, 1953), Vols. 1 and 2.

[28] E. G. Kalnins, W. Miller, and P. Winternitz, SIAM J. Appl. Math. 30, 630 (1976).

[29] R. D. Spence, Am. J. Phys. 27, 329 (1959).

[30] C. A. Coulson and P. D. Robinson, Proc. London Phys. Soc. 71, 815 (1958).

[31] D. Park, Z. Phys. 159, 155 (1960).

[32] K. Helfrich, Theor. Chim. Acta 24, 271 (1972); G. J. Hatton, Phys. Rev. A 16, 1347 (1977).

[33] H. G. Kuhn, Atomic Spectra, 2nd ed. (Academic, New York, 1969). 